\title{
Infección del tracto genital superior femenino: Revisión pictográfica por tomografía computada según clasificación clínica
}

\author{
Dres. Giancarlo Schiappacasse $F^{(1)}$, Eric Gana $G^{(2)}, M$. Ignacia Ríos $P^{(2)}$, Pablo Soffia $S^{(1)}$.
}

1. Médico Radiólogo. Facultad de Medicina Universidad del Desarrollo - Clínica Alemana de Santiago. Chile.

2. Interno Universidad del Desarrollo Santiago. Santiago - Chile.

Infection of the female upper genital tract: Pictorial review by computed tomography according to clinical classification

Abstract: Upper genital tract infection (UGTI) or female pelvic inflammatory disease (PID) comprises a number of inflammatory conditions of the female reproductive tract. The clinical presentation is varied and most occur as a lower quadrant abdominal pain or pelvic pain, and may even be diffuse and often manifest as an acute abdominal pain difficult to diagnose. The clinical presentation and anatomic involvement are varied. In 1982 Monif designed a classification based on the anatomical commitment of the affected structures, from a localized infection such as salpingitis, to more serious conditions such as life-threatening ruptured tubo-ovarian abscess (TOA). Images together with case history form a fundamental basis for the diagnosis and so define therapeutic management. In the following article a series of cases are presented with their main findings to the computed tomography (CT) classified according to Monif's staging.

Keywords: Computed tomography (CT), Monif stadiums, Pelvic inflammatory disease (PID).

Resumen: La infección del tracto genital superior (ITGS) femenino o enfermedad inflamatoria pélvica (EIP) comprende una serie de condiciones inflamatorias del tracto reproductivo femenino. La presentación clínica es variada y la mayoría se presenta como un dolor abdominal de cuadrantes inferiores o dolor pélvico, pudiendo ser incluso difuso y muchas veces manifestarse como un dolor abdominal agudo de difícil diagnóstico. Las formas de presentación clínica y el compromiso anatómico son variados. En 1982 Monif diseñó una clasificación que se basa en el compromiso anatómico de las estructuras afectadas, desde una infección localizada como salpingitis, hasta cuadros más graves con riesgo vital como un absceso tubo ovárico (ATO) roto. Las imágenes junto a la clínica forman un pilar fundamental para el diagnóstico y así definir una conducta terapéutica. En el siguiente artículo se presenta una serie de casos con sus principales hallazgos a la tomografía computada (TC) clasificados por los estadios de Monif. Palabras claves: Enfermedad inflamatoria pélvica (EIP), Estadios de Monif, Infección del tracto genital superior (ITGS), Tomografía computada (TC).

Schiappacasse G, et al. Infección del tracto genital superior femenino: Revisión pictográfica por tomografía computada (TC) según clasificación clínica. Rev Chil Radiol 2014; 20(1): 31-37.

Correspondencia: Giancarlo Schiappacasse / gschiappacasse@gmail.com

Trabajo recibido el 29 de agosto de 2013. Aceptado para publicación el 26 de febrero de 2014.

\section{Introducción}

La infección del tracto genital superior (ITGS) es una condición común entre las mujeres en edad reproductiva ${ }^{(1)}$. Corresponde a la infección y la inflamación resultante del tracto superior de los órganos reproductivos femeninos, incluyendo el endometrio, las trompas de Falopio y los ovarios ${ }^{(2)}$. Reportes de la literatura estiman que es una de las enfermedades más comunes del género femenino afectando 1 millón de mujeres, representando 275.000 hospitalizaciones al año en EE.UU ${ }^{(3,4)}$. En Chile, en el Servicio de Ginecología del Hospital San Borja Arriarán, 2,4\% de los egresos en 1991 fueron por esta causa ${ }^{(5)}$, en otros reportes cerca del $39 \%$ de las pacientes afectadas por EIP correspondían a ATO la cual ha aumentado especialmente en mujeres mayores de 40 años ${ }^{(6)}$.

La ITGS usualmente es causada por una infección de tipo ascendente, con etiología bien esclarecida por la literatura donde sus principales agentes involucrados son Neisseria gonorrhoeae o Chlamydia trachomatis, aunque entre un $30 \%-40 \%$ de los casos es de causa polimicrobiana $^{(7)}$.

Entre los factores de riesgo para EIP se incluyen mujeres jóvenes, múltiples parejas sexuales, alta frecuencia coital, bajo nivel socioeconómico, duchas vaginales y el uso de dispositivo intrauterino (DIU) (sobre todo durante 
los primeros meses después de la inserción), así como otras formas de instrumentación pélvica ${ }^{(2)}$.

Dentro de las complicaciones secundarias a una ITGS destacan las derivadas por daño de la trompa uterina, cicatrices y oclusión ${ }^{(2)}$. Los pacientes que han cursado con una ITGS tienen seis veces mayor riesgo de embarazo ectópico, mayor riesgo de futuros episodios de EIP; además un $20 \%$ de los pacientes con una historia de ITGS se quejará de dolor pélvico crónico(1). Dentro de las complicaciones más graves se encuentra la infertilidad asociada a una obstrucción de las trompas de Falopio que ocurrirá en el $8 \%$ de los pacientes después de un solo episodio de ITGS, con un aumento de hasta un $20 \%$ después de dos episodios de ITGS y hasta un $40 \%$ después de tres episodios ${ }^{(3)}$.

Debido a la variedad de complicaciones que se producen al corto y largo plazo es importante tener un diagnóstico precoz y certero de una ITGS, para lograr de esta manera un tratamiento oportuno. Sin embargo la clínica presentada es muchas veces variada y poco específica presentando síntomas como fiebre, dolor abdominal o dolor pélvico, flujo vaginal, metrorragia, dispareunia, disuria, sensibilidad anexial o cervical, náuseas, vómitos, y otros vagos síntomas constitucionales ${ }^{(3,7,8)}$. Por otro lado, la literatura describe que hasta un $35 \%$ de los pacientes con EIP son asintomáticas ${ }^{(2)}$.

La laparoscopía ha sido durante mucho tiempo el estándar de referencia en el diagnóstico definitivo de ITGS ${ }^{(9,10)}$, sin embargo, ésta requiere de anestesia además de ser un procedimiento invasivo que puede dar lugar a complicaciones ${ }^{(9)}$. Esta es preferida en las pacientes con moderado o severo dolor pélvico.

En los EE.UU., la ecografía transvaginal es un procedimiento de rutina utilizado en paciente con dolor pélvico ${ }^{(11)}$. Estudios anteriores han demostrado que la ecografía transvaginal ha obtenido buenos resultados en el diagnóstico de EIP cuando los hallazgos incluyen trompas uterinas llenas de líquido y/o engrosadas ${ }^{(12)}$.

Por otra parte la TC ha sido usada en el diagnóstico de abscesos de complejo tubo ováricos ${ }^{(13)}$ y ha sido de gran utilidad en cuadros clínicos de EIP de difícil diagnóstico clínico-ecográfico ${ }^{(14,15)}$, siendo en muchas ocasiones la herramienta diagnóstica.

El presente artículo tiene como objetivo desarrollar una revisión pictográfica que evalúa los distintos estadios clínicos de la EIP, según la clasificación de Monif propuesta en $1982^{(16)}$, descritos en la tabla I y su correlación con TC multislice, analizando los principales hallazgos en imágenes de cada uno.

\section{Tabla I.}

Estadio I Salpingitis aguda sin peritonitis

Estadio II Salpingitis aguda con peritonitis

Estadio III Salpingitis con absceso tubo ovárico

Estadio IV ATO roto

\section{Estadio I: Salpingitis aguda sin peritonitis}

Se produce una infección e inflamación ascendente de las trompas uterinas, con edema.

El estudio por TC es generalmente negativo, sin embargo, se pueden encontrar alteraciones como mínima cantidad de líquido libre en el fondo de saco recto-uterino; aumento de volumen del ligamento útero-sacro; aumento de densidad del tejido adiposo adyacente; engrosamiento de las paredes de las trompas uterinas; endometrio engrosado con líquido en su interior; $y$ el útero puede perder nitidez de sus contornos y límites con los anexos ${ }^{(2)}$. Los ovarios pueden estar aumentados de tamaño y perder diferenciación córtico-medular ${ }^{(17)}$. El objetivo en este estadío es tratar la infección ${ }^{(18)}$.

Caso clínico: Paciente de 44 años, sin antecedentes mórbidos. Usuaria de DIU, consulta por cuadro de 3 días de evolución de dolor abdominal hipogástrico, irradiado a fosa lumbar derecha, asociado a fiebre, síntomas urinarios, náuseas y vómitos. Consulta al servicio de urgencia hemodinámicamente estable, el abdomen se encuentra sensible, sin signos de irritación peritoneal. Se realiza TC de abdomen y pelvis contrastado, que muestra salpingitis aguda izquierda.
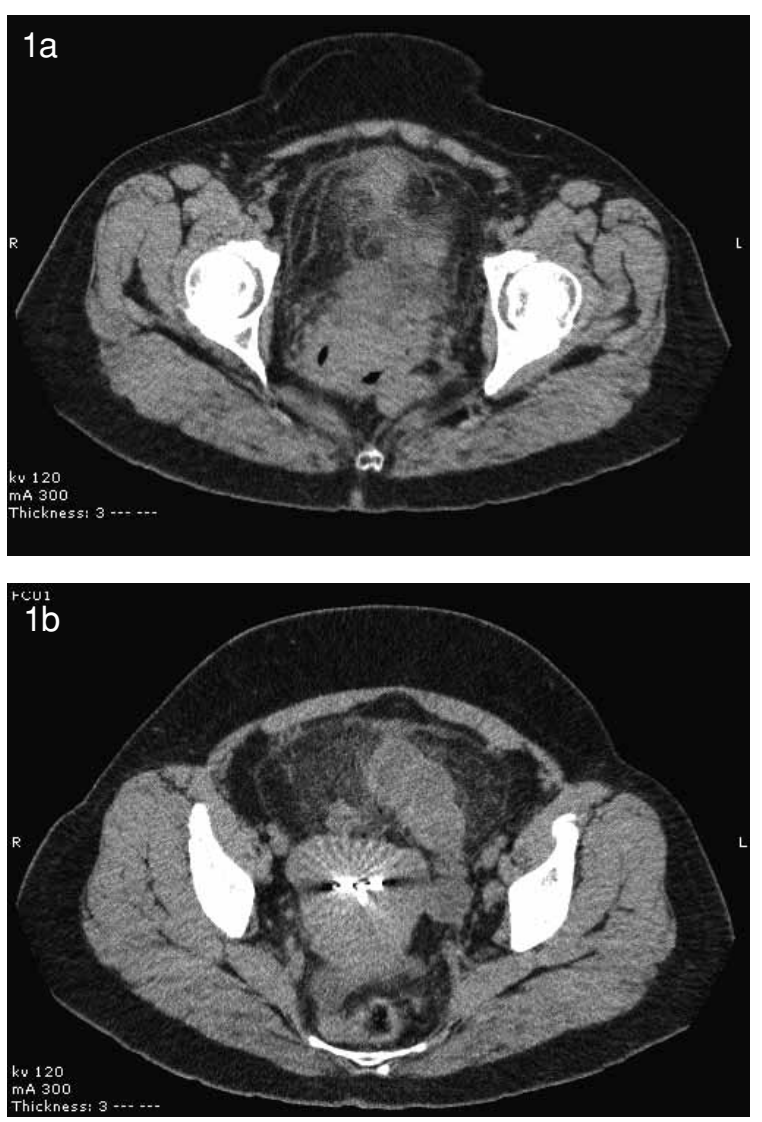

Figura 1. Salpingitis aguda sin signos de peritonitis. Masa quística en la cavidad pelviana dependiente del anexo izquierdo, de contornos lobulados y aspecto tubular, asociado a un aumento de la densidad de la grasa adyacente. No se evidencian colecciones líquidas. Útero de tamaño conservado con DIU in situ. 
Caso clínico: Paciente de 40 años, Consulta por cuadro de pocas horas de evolución de dolor abdominal en fosa ilíaca derecha, sin blumberg. Acude febril y hemodinámicamente estable. Al examen físico destaca dolor a la palpación pélvica baja. Se realiza TC de abdomen y pelvis contrastado que muestra salpingitis aguda derecha.
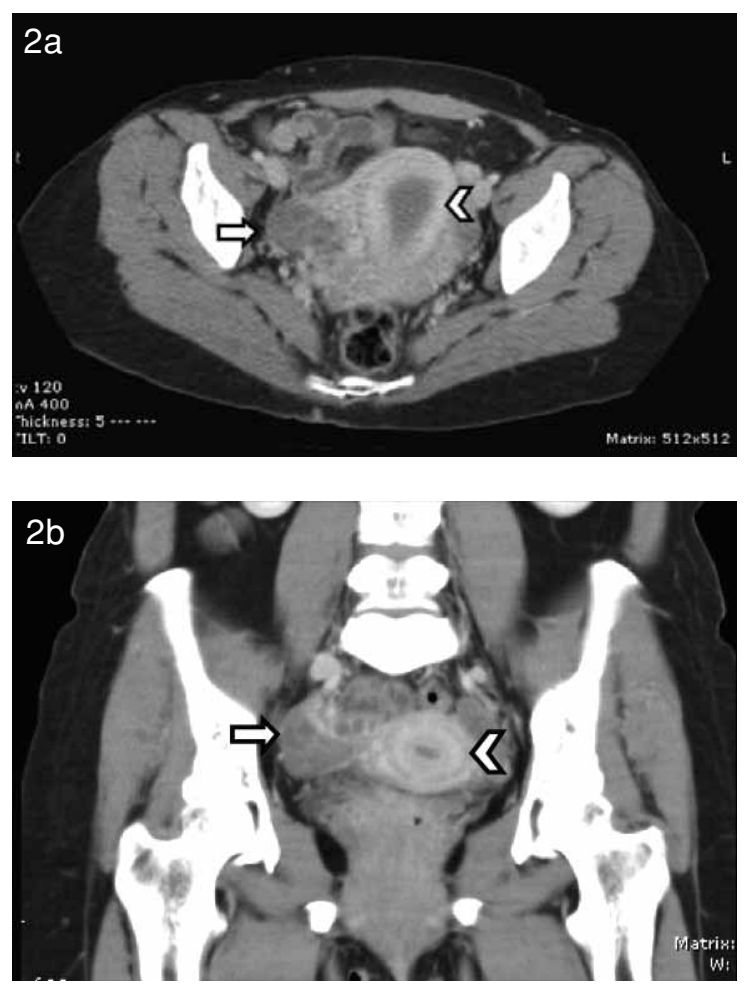

Figura 2. Salpingitis aguda derecha (Flechas). Tanto en corte axial como en coronal se identifica, en relación al anexo derecho, una estructura tubular de paredes hipercaptantes con contenido hipodenso, correspondiente a salpingitis (Cabezas de flecha). Se evidencia en ambos cortes, útero aumentado de tamaño, de paredes engrosadas $e$ hipercaptantes, con contenido hipodenso en su interior, hallazgos que sugieren endometritis.

Caso clínico: Paciente 32 años, usuaria de DIU consulta por cuadro de dolor abdominal difuso, acude febril. Al examen físico el abdomen se encuentra distendido, con dolor a la palpación pélvica, sin blumberg, se solicita TC de abdomen y pelvis con contraste.

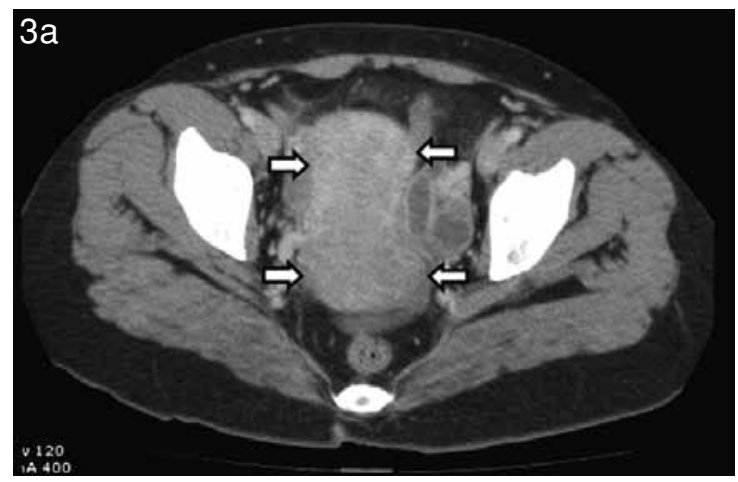

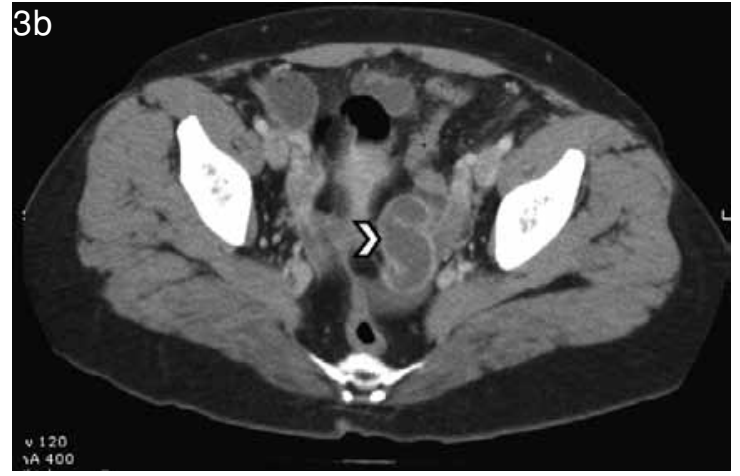

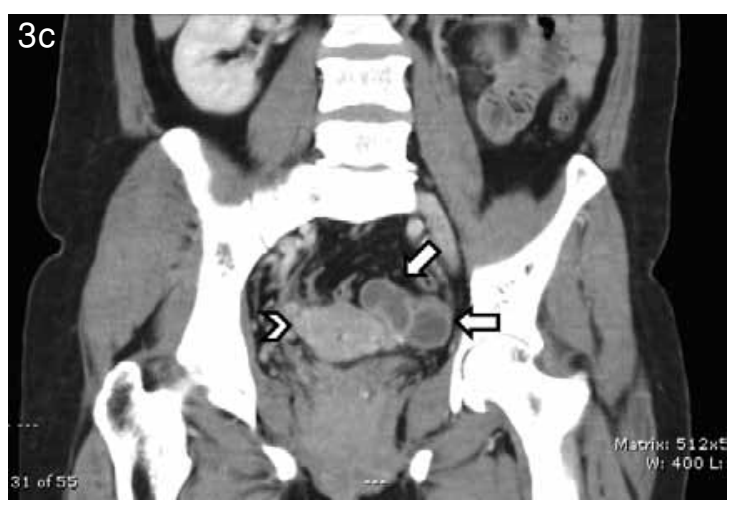

Figura 3. Salpingitis aguda sin peritonitis. Se aprecian cambios inflamatorios de la grasa pélvica en $a, b$ y $c$. a) Heterogeneidad de la densidad uterina, con aumento de la captación y engrosamiento parietal, de bordes mal definidos, aumento de tamaño, signos que sugieren endometritis (Flechas). b) Imágenes hipodensas de aspecto tubular con realce por contraste de la pared, de forma serpentiginosa correspondiente a la tuba uterina, con contenido líquido y cambios inflamatorios (Cabeza de flecha). c) Masa quística en relación a anexo izquierdo con compromiso de la grasa adyacente y desplazamiento de estructuras en relación a dicho anexo (Flechas), se aprecia compromiso uterino descrito en corte coronal (Cabeza de flecha).

\section{Estadio II: Salpingitis aguda con peritonitis}

Se produce debido a un proceso evolutivo de la ITGS que no ha recibido tratamiento. El objetivo de este estadio es conservar la fertilidad ${ }^{(18)}$. En el estudio por imágenes se puede encontrar líquido libre en fondo de saco rectouterino o abscesos con tabicaciones ${ }^{(1)}$.

Caso clínico: Paciente de 39 años, consulta por cuadro de 24 horas de evolución de dolor abdominal difuso, tipo cólico, asociado a distensión abdominal y náuseas, sin eliminación de deposiciones ni gases por ano. Al examen físico se evidencia abdomen distendido y sensible a la palpación, blumberg positivo. Se realiza TC de abdomen y pelvis contrastado que muestra salpingitis aguda izquierda con pelviperitonitis secundaria, con compromiso por contigüidad del colon sigmoides y una pequeña colección en fondo de saco rectouterino. 

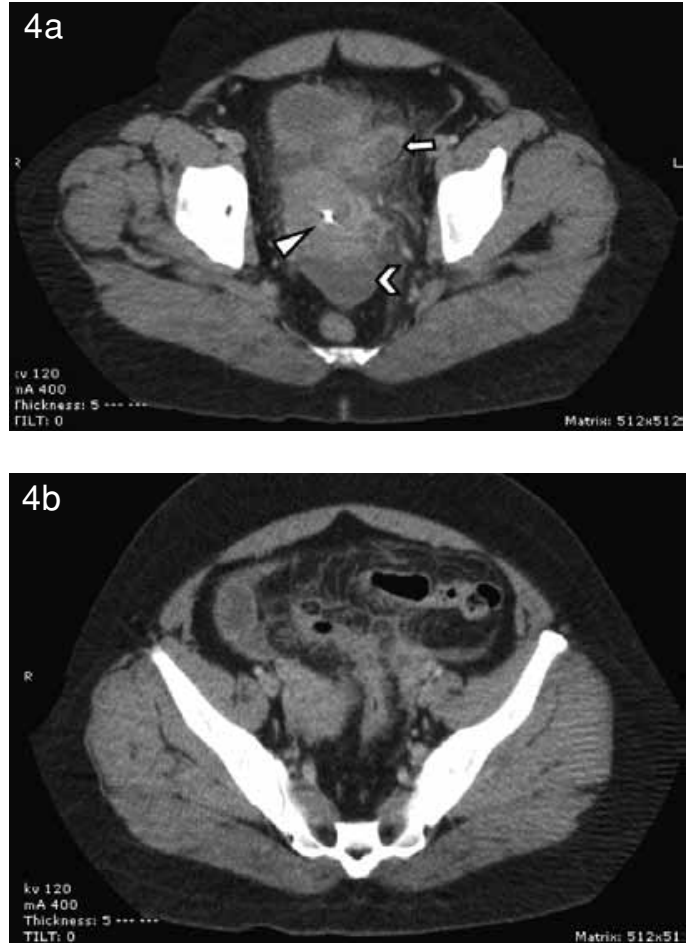

Figura 4. Salpingitis aguda con peritonitis. a) Imagen tubular de paredes hipercaptantes adyacente al cuerno uterino izquierdo, sugerente de salpingitis aguda (Flecha). Imagen hipodensa que desciende hacia el fondo de saco rectouterino, compatible con una colección de 6 × $4 \mathrm{~cm}$ (Cabeza de flecha). Imagen de DIU in situ (Triángulo). b) Aumento de la densidad de la grasa adyacente y cambios inflamatorios parietales del colon sigmoides por contigüidad. Ovarios de características normales.

Caso clínico: Paciente de 44 años, que consulta por cuadro clínico de abdomen agudo. Se realiza TC de abdomen y pelvis que muestra pelviperitonitis de origen anexial izquierdo y DIU in situ.

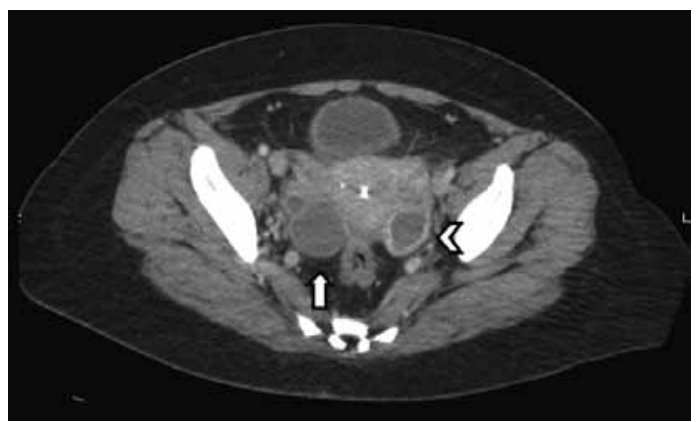

Figura 5. Salpingitis aguda con peritonitis. Útero con DIU in situ. Imagen focal hipodensa de aspecto quístico en relación a anexo derecho (Flecha). Anexo izquierdo de paredes engrosadas e hipercaptantes, con cambios inflamatorios de la grasa local (Cabeza de flecha).

Caso clínico: Paciente de 42 años, consulta por cuadro de varios días de dolor en región lumbar izquierda. Se realiza TC de abdomen y pelvis que muestra pelviperitonitis secundaria a proceso inflamatorio de origen anexial bilateral.
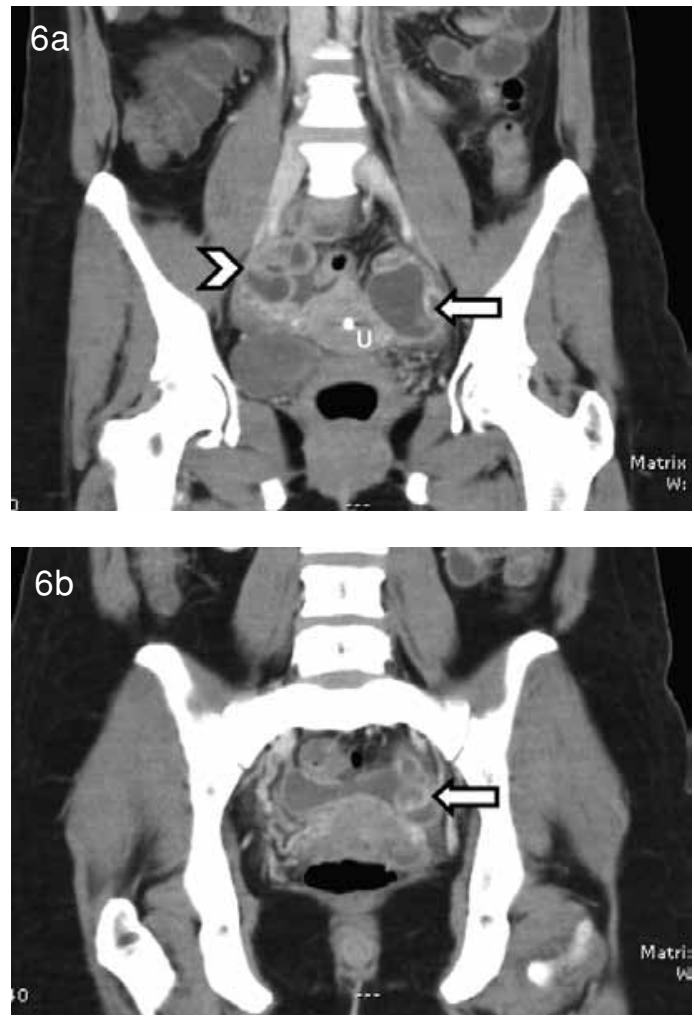

Figura 6. Salpingitis con peritonitis. a) A nivel de la pelvis, en relación al anexo izquierdo, se observa imagen sólido quística, de bordes poco precisos, que realza de forma heterogénea con contraste. Dilatación tubular de paredes engrosadas, que realzan con contraste en relación a anexo y tuba derecha (Cabeza de flecha). Colección de aproximadamente $6 \mathrm{~cm}$ de diámetro en contacto con la pared superior del útero $y$ cambios inflamatorios difusos del mesenterio adyacente (Flecha). Útero con DIU in situ (U). b) Heterogeneidad de captación de contraste en pared superior del útero. Se aprecia dilatación de la tuba uterina izquierda con paredes engrosadas (Flecha) y cambios inflamatorios difusos de la grasa en a y $b$.

\section{Estadio III: Salpingitis con formación de ATO}

La progresión de la infección e inflamación determinan destrucción de las estructuras anatómicas normales, con la formación de una masa inflamatoria que involucra tanto al ovario como a la tuba uterina. El tratamiento por lo general es médico, sin embargo, existen indicaciones de cuándo es necesaria la resolución quirúrgica ${ }^{(3,7)}$.

Se produce liberación de contenido purulento a la cavidad peritoneal con inflamación de las vísceras contiguas y aglutinación posterior de éstas, conformando el complejo tubo ovárico que involucra: tubas, ovarios, ligamento ancho, intestino y epiplón ${ }^{(19)}$.

Las indicaciones quirúrgicas pueden ser inmediatas o mediatas. Dentro de las inmediatas se describe un tumor mayor a $8 \mathrm{~cm}$, mala respuesta a antibióticos luego de 72 horas o shock séptico. Dentro de las mediatas se describe un tumor que no disminuye más de $50 \%$ de tamaño en 6 meses y algia pélvica crónica ${ }^{(20)}$. El objetivo del tratamiento en este estadio es conservar el ovario ${ }^{(18)}$.

Los hallazgos en la TC incluyen, masa anexial sólido 
quística con realce de contraste de la pared, dilatación de las tubas uterinas con edema de pared e hipercaptación del contraste, de aspecto serpentiginoso, alteración de la grasa y compromiso inflamatorio de órganos adyacentes, engrosamiento de ligamentos uterinos con el uso de contraste y gas en tubas uterinas, que a pesar de ser un signo muy específico de ATO; es un hallazgo inusual(8,21,22).

Caso clínico: Paciente de 20 años, con antecedentes de múltiples cirugías urogenitales. Consulta por cuadro de 2 días de evolución de dolor abdominal hipogástrico y en fosa ilíaca derecha. Se realiza TC de abdomen y pelvis que muestra absceso tubo ovárico derecho, neovejiga y leve hidroureteronefrosis bilateral.
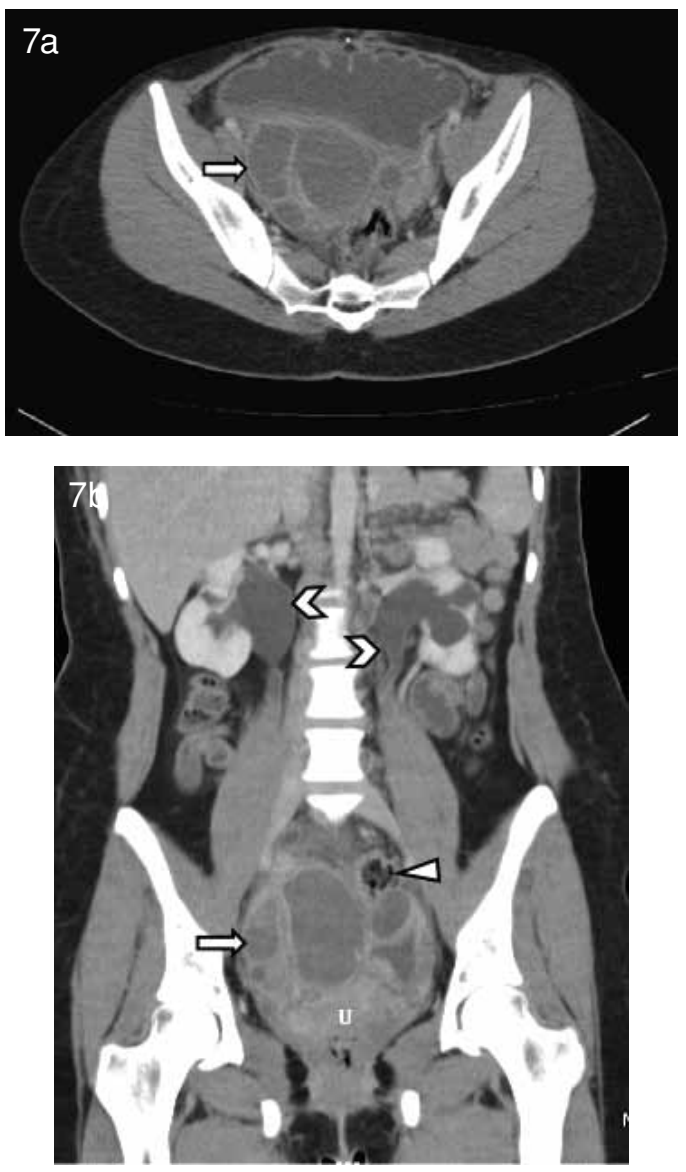

Figura 7. Salpingitis con formación de ATO. a) Flecha. En la región anexial derecha se observa masa quística compleja con componente tubular serpentiginoso, que corresponde a una dilatación patológica de la tuba uterina. Dicha formación quística muestra realce capsular por contraste y alcanza un diámetro de $7.5 \mathrm{~cm}$. b) Flecha. En hemipelvis derecha, en relación a anexo derecho, se observa formación quística que presenta realce de la pared, de contenido homogéneo, que desplaza parcialmente vejiga hacia la izquierda. Cabezas de Flecha. Hidroureteronefrosis, se observa dilatación de la pelvis renal y uréter proximal bilateral. Triángulo. Relación de masa anexial con asas de intestino grueso, el cual presenta paredes engrosadas y compromiso inflamatorio de grasa periférica. (U) Útero desplazado hacia izquierda, con heterogeneidad de la densidad y bordes mal definidos. a y b. Grasa perilesional presenta heterogeneidad en su densidad indicando cambios inflamatorios importantes.
Caso clínico: Paciente de 41 años, consulta por cuadro de un mes de evolución de dolor en flanco izquierdo, acude con $\mathrm{T}^{\circ} 37,4^{\circ} \mathrm{C}$ y hemodinámicamente estable. Al examen destaca abdomen doloroso en fosa ilíaca izquierda, sin signos de irritación peritoneal, sin masas palpables. Se realiza TC de abdomen y pelvis que muestra absceso tubo ovárico izquierdo y colección en fondo de saco rectouterino.
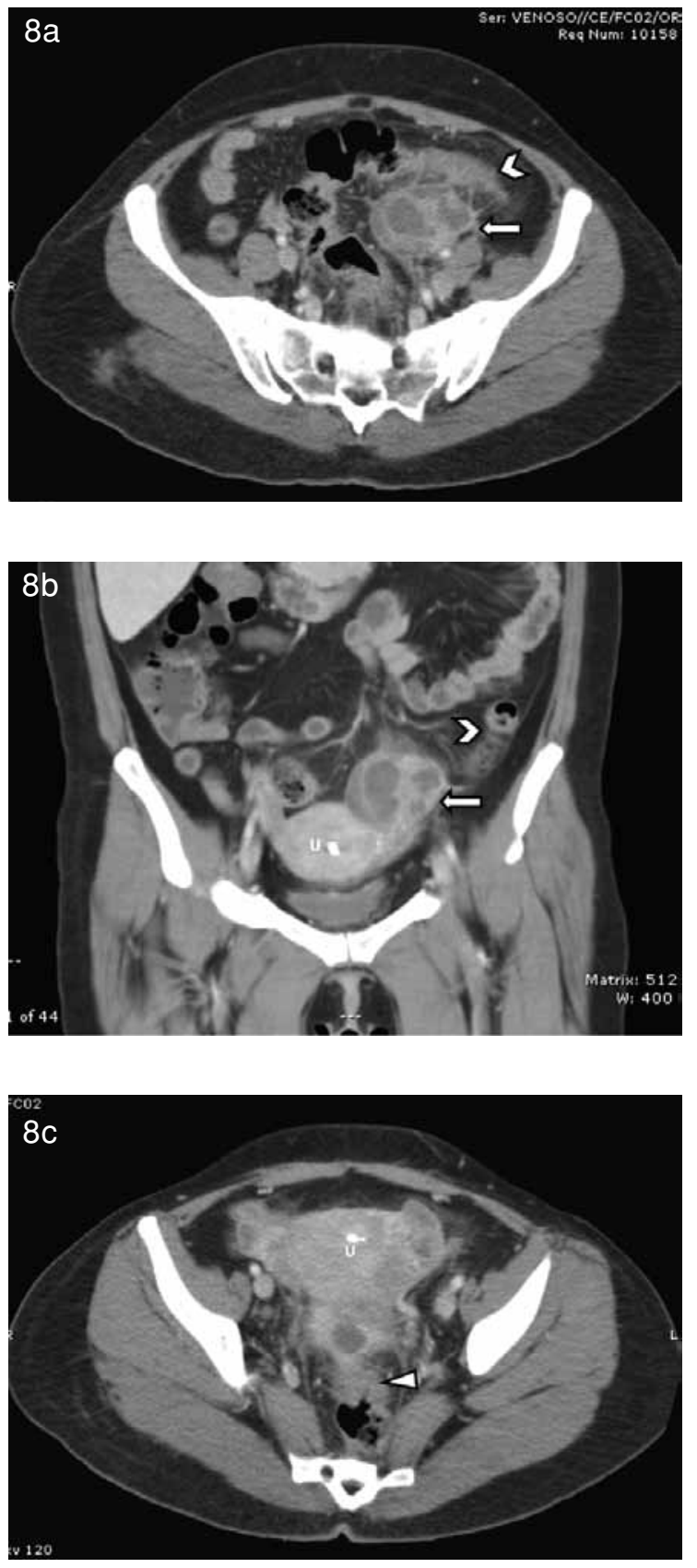

Figura 8. Salpingitis con formación de ATO. Flechas. Aumento de volumen del anexo izquierdo, con múltiples abscesos en su interior (Cabezas de Flecha). Cambios inflamatorios de la tuba uterina izquierda y grasa del mesosigmoides, con engrosamiento parietal reactivo de éste. Triángulo. Adyacente al anexo, en el fondo de saco rectouterino, se observa una pequeña colección. U. Útero en anteroversoflexión con DIU in situ. 


\section{Estadio IV: Absceso tubo ovárico roto}

Estadio de gran mortalidad, sobre todo cuando el diagnóstico es tardío. Se debe sospechar por un cambio en el cuadro clínico de una paciente cursando una $\mathrm{EIP}^{(20)}$. Generalmente el examen físico es poco concluyente, pudiendo encontrarse abdomen levemente distendido, reducción de ruidos hidroaéreos y signos de irritación peritoneal, como aumento de la resistencia muscular con eventual Blumberg ${ }^{(23)}$.

El manejo es de urgencia con laparotomía exploratoria, lavado generoso de la cavidad abdomino-pélvica y resección de los órganos comprometidos ${ }^{(24)}$.

El objetivo del tratamiento en este estadio es salvar la vida de la paciente ${ }^{(18)}$.

Caso clínico: Paciente de 39 años, usuaria de DIU cambiado hace un mes. Consulta por cuadro de 20 días de dolor abdominal, inicialmente hipogástrico y luego difuso, asociado a sensación febril, calofríos, CEG, náuseas y vómitos. Ingresa en shock séptico compensado. Se realiza TC de abdomen y pelvis que muestra múltiples abscesos tubo ováricos bilaterales, con signos de peritonitis.
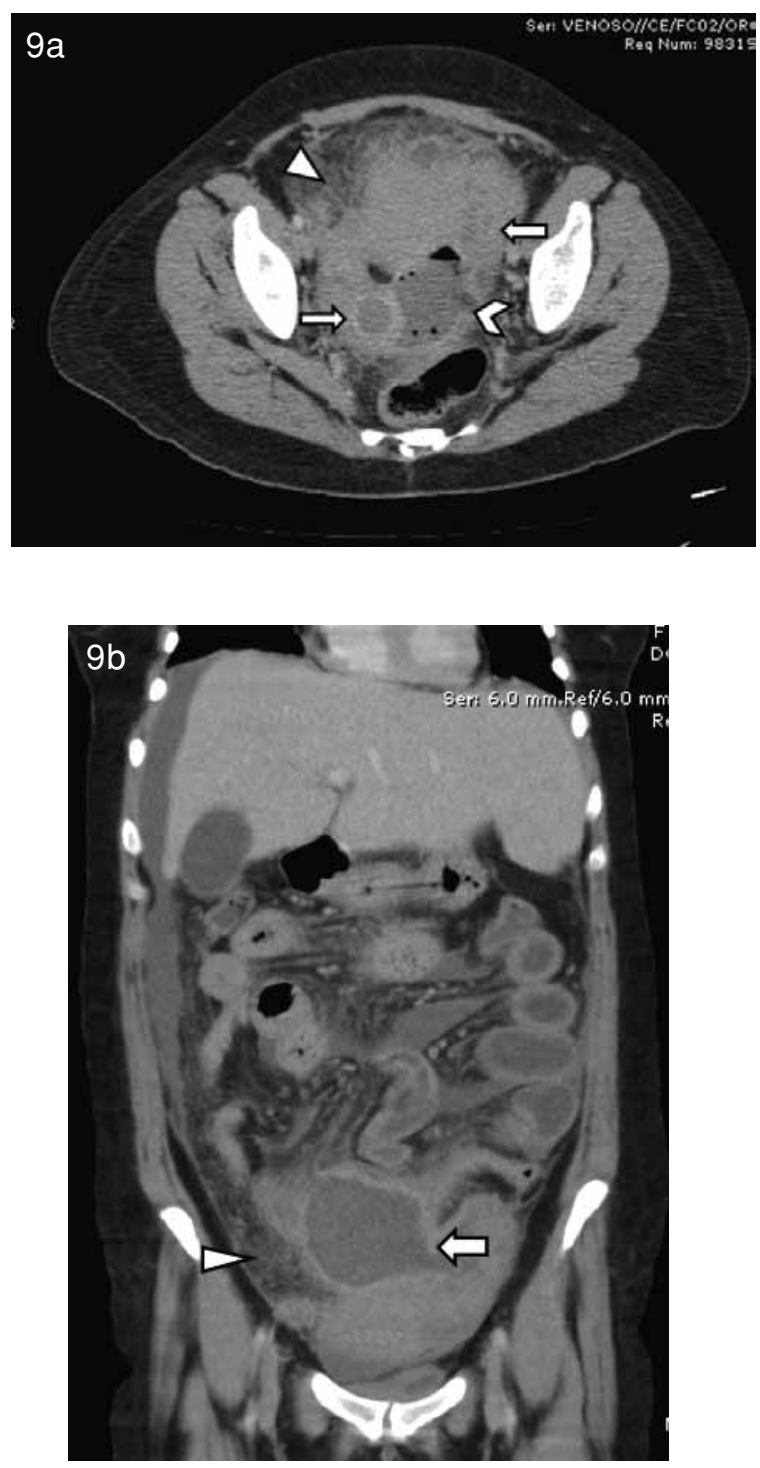

Caso clínico: Paciente de 27 años. Consulta por cuadro de pocas horas de evolución de dolor abdominal, asociado a náuseas. Ingresa hemodinámicamente estable y febril. Se realiza TC de abdomen y pelvis con contraste que muestra pelviperitonitis secundaria a ATO roto.
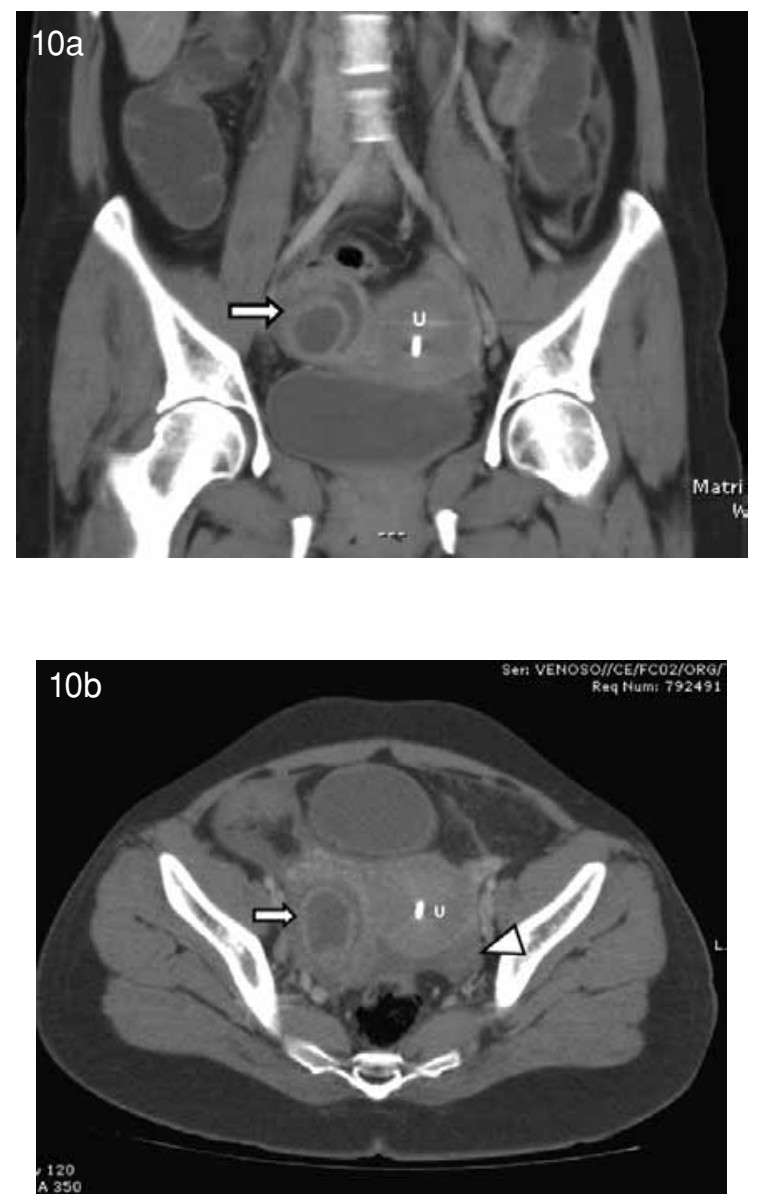

Figura 10. Absceso tubo ovárico roto. Se aprecian asas de intestino delgado dilatadas con leve engrosamiento parietal. $U$. Útero con DIU in situ (Flechas). Anexo derecho aumentado de tamaño, de $6 \mathrm{~cm}$ de diámetro mayor, heterogéneo, con áreas quísticas en su espesor y realce capsular por contraste. Triángulo. Aumento de la densidad de la grasa pelviana y leve cantidad de líquido libre intraperitoneal.
Figura 9. Absceso tubo ovárico roto. Útero en anteroversión. (Flechas). Ambos anexos se encuentran reemplazados por múltiples colecciones líquidas de paredes hipercaptantes, compatible con abscesos (Cabeza de flecha). Se aprecia nivel hidroaéreo dentro de cavidad, signo muy sugerente de ATO roto (Triángulos). Se aprecia gran alteración de grasa perilesional de componente inflamatorio y líquido libre peritoneal. 


\section{Conclusión}

La presentación de una ITGS puede ser variada dependiendo del compromiso anatómico y la gravedad del cuadro clínico.

La ITGS es uno de los múltiples diagnósticos diferenciales de abdomen agudo. Como estudio imagenológico inicial se recomienda el uso de la ultrasonografía transvaginal, siendo ésta a veces insuficiente para clarificar diagnóstico, es en estas condiciones cuando la TC de abdomen y pelvis es de gran utilidad. Es importante mencionar que actualmente el uso de la resonancia nuclear magnética posee mayor sensibilidad y especificidad que la TC, además de ser menos riesgosa por no utilizar radiación ionizante, en especial en mujeres jóvenes.

El claro diagnóstico por imágenes y su correlación clínica es de suma importancia, debido a que la presentación y gravedad de la ITGS no guarda directa relación con los hallazgos encontrados en las imágenes, es decir, cuadros de abdomen agudo febriles pueden ser la expresión clínica tanto de una salpingitis como de un ATO.

Cuando se sospecha una ITGS, la TC de abdomen y pelvis, además de ser una herramienta de gran utilidad para el diagnóstico, permite clasificar con mayor exactitud el estadio clínico, es decir, el compromiso anatómico en el que se encuentra la enfermedad, lo cual influiría en la gravedad y la decisión terapéutica por parte del tratante.

\section{Bibliografía}

1. Timo A. Tukeva, Hannu J. Aronen, Pertti T. Karjalainen, et al. MR Imaging in Pelvic Inflammatory Disease: Comparison with Laparoscopy and US. Radiology 1999; 210: 209-216.

2. Joseph W. Sam, Jill E. Jacobs, Bernard A. Birnbaum, et al. Spectrum of CT Findings in Acute Pyogenic Pelvic Inflammatory Disease. RadioGraphics 2002; 22: 1327-1334.

3. McCormack WM. Pelvic Inflammatory disease. N Engl J Med 1994; 330: 115-119.

4. Quiroz FA. Pelvic Inflammatory disease. Appl Radiol 1999; 28: 30-35.

5. Martínez MA. Vaginitis and pelvic inflammatory prevention. Medwave 2007 Mar; 7(2): e1265 doi: 10.5867/ medwave.2007.02.1265.

6. Ovalle A, Casanova A, Kakarieka E, De Jourdan F, et al. Epidemiología, Resultados clínicos y costos del tratamiento del abceso tuvoovárico, en un hospital público de Santiago. Rev Chil Obstet Ginecol 2008; 73: 374-380.

7. Soper DE. Pelvic inflammatory disease. Infect Dis Clin N Amer 1994; 8: 821-840.

8. Quiroz FA. Pelvic inflammatory disease. Appl Radiol 1999; 28: 30-35.

9. Jacobson L, Westrom L. Objectivized diagnosis of acute pelvic inflammatory disease: diagnostic and prognostic value of routine laparoscopy. AmJ Obstet Gynecol 1969; 105: 1088-1098.

10. Sellors J, Mahony J, Goldsmith C, et al. The accuracy of clinical findings and laparoscopy in pelvic inflammatory disease. Am J Obstet Gynecol 1991; 164: 113-120.

11. Paavonen J, Aine R, Teisala K, et al. Chlamydial endometritis. J Clin Pathol 1985; 38: 726-732.

12. Cacciatore B, Leminen A, Ingman Friberg $S$, et al. Transvaginal sonographic findings in ambulatory patients with suspected pelvic inflammatory disease. Obstet Gynecol 1992; 80: 912-916.

13. Wilbur AC, Aizenstein RI, Napp TE. CT findings in tuboovarian abscess. AJR 1992: 158: 575-579.

14. Urban BA, Fishman EK. Spiral CT of the female pelvis: clinical applications. Abdom Imaging 1995; 20: 9-14.

15. Romo LV, Clarke PD. Fitz-Hugh-Curtis syndrome: pelvic inflammatory disease with unusual CT presentation. $J$ Comput Assist Tomogr 1992; 16: 832-833.

16. Monif G. Clinical Staging of acute bacterial salpingitis and its therapeutic ramifications. Am J Obstet Gynecol 1982; 143: 489-495.

17. Cacciatore B, Leminen A, Ingman-Friberg S, et al. Transvaginal sonographic findings in ambulatory patients with suspected pelvic inflammatory disease. Obstet Gynecol 1992; 80: 912-916.

18. Guilles R. G. Monif. Enfermedades infecciosas en Obstetricia y Ginecología. Fases de Gainesville de la salpingitis aguda. La Habana: Salvat; 1985. p. 535.

19. Siu, Alejandro. Conceptos, diagnósticos y tratamiento del plastrón anexial y absceso tubo-ovárico no roto. Diagnostico 1990; 25: 5-6.

20. Sergio Silva S. Infecciones en Ginecología y Obstetricia. Chile: Mediterráneo; 1997.

21. Ellis JH, Francis IR, Rhodes M, Kane NM, et al. CT findings in tuboovarian abscess. J Comput Assist Tomogr 1991; 15: 589-592.

22. Wilbur AC, Aizenstein RI, Napp TE. CT findings in tuboovarian abscess. AJR Am J Roentgenol 1992; 158: 575-579.

23. Pedowitz P, Bloomfield RD. Ruptured adnexal abscerss (tuboovarian) with generalized peritonitis. Am J Obstet Gynecol 1964; 88: 721.

24. Granberg S, Gjelland K, Ekerhovd E. The management of pelvic abscess. Best Pract Res Clin Obstet Gynaecol 2009; 23: 667. 